\title{
Théories du changement : comment élaborer des modèles utiles
}

\author{
John Mayne \\ Ottawa (Ontario)
}

\begin{abstract}
Résumé : Bien que les théories du changement soient souvent l'objet d'articles en évaluation et qu'il y ait un consensus sur ce qu'est, conceptuellement, une théorie du changement, il n'y a, en réalité, au-delà des principes généraux, que peu de consensus sur ce quelles comprennent, sur ce qu'elles devraient mettre en valeur ainsi que de la manière dont elles devraient être représentées et utilisées. Cet article donne un aperçu de certains modèles de théories du changement qui se sont révélés utiles, tant pour des interventions simples que complexes, et présente la façon dont ils ont été developpés. Les modèles sont intuitifs et flexibles, leurs composantes sont bien définies et ils s'appuient sur des modèles de causalité rigoureux. Ces modèles fournissent un cadre structuré pour lélaboration de théories utiles du changement et pour lanalyse de l'intervention.
\end{abstract}

Mots clés : liens de causalité, intervention complexe, chaîne d'effets, modèle logique, chaîne des résultats, théorie du changement, théorie de la portée.

\begin{abstract}
Although theories of change are frequently discussed in the evaluation literature and there is general agreement on what a theory of change is conceptually, there is actually little agreement beyond the big picture of just what a theory of change comprises, what it shows, how it can be represented, and how it can be used. This article outlines models for theories of change and their development that have proven quite useful for both straightforward and more complex interventions. The models are intuitive, flexible, and well-defined in terms of their components, and they link directly to rigorous models of causality. The models provide a structured framework for developing useful theories of change and analyzing the intervention they represent.
\end{abstract}

Keywords: causal links, complex intervention, impact pathways, logic model, results chain, theory of change, theory of reach

Les modèles décrivant la logique des interventions sont fréquemment analysés et utilisés dans le domaine de lévaluation. Voir, par exemple, Patton (2008); Chen (2015); Rossi, Lipsey et Freeman (2004); Morra Imas et Rist (2009); Funnell et

Auteur-ressource : John Mayne, john.mayne@rogers.com

Remerciement : L'équipe éditoriale souhaite remercier Richard Marceau et Benoît Gauthier, qui ont tous deux révisé la traduction de cet article.

C 2017 Canadian Journal of Program Evaluation / La Revue canadienne d'évaluation de programme 32.2 (Fall / automne ), 174-201 doi: 10.3138/cjpe.31144 
Rogers (2011). Suchman (1967) et Bickman (1987) ont été parmi les premiers à se pencher sur la question. Toutefois, les termes employés pour décrire ces modèles sont très variés; on parle notamment de théorie du programme, de modèle logique, de théorie du changement, de chaîne des résultats ou d’effets, de théorie de l'action, de théorie de l'implantation, et il ne semble pas y avoir de consensus sur la terminologie ou le sens des termes. Funnell et Rogers (2011, p. 15-34), tout comme Patton (2008, p. 336-340), ont d'ailleurs discuté de leurs spécificités et présenté leur histoire.

J'emploierai ici le terme théorie du changement. Les théories du changement peuvent être utilisées de façon très variée, tant pour le développement, la gestion et l'évaluation des interventions. Mayne et Johnson (2015) discutent de l'utilisation des théories de changement pour:

\section{La conception et la planification des interventions}

1. Conception des interventions

2. Compréhension et représentations communes des interventions par les parties prenantes

3. Identification des enjeux en lien avec l'équité, le genre et les inégalités de pouvoir (empowerment issues)

4. Évaluation ex ante des interventions proposées

\section{La gestion des interventions}

5. Conception de systèmes de monitorage

6. Compréhension de la mise en œuvre, gestion adaptative et apprentissage

\section{L'évaluation des interventions}

7. Conception des questions, méthodes et outils d'évaluation

8. Formulation des hypothèses pour la production des effets

9. Production de rapports de performance

\section{La transférabilité}

10. Généralisation théorique, à d'autres contextes et à des échelles différentes.

Dans la cinquième partie de leur ouvrage, Funnell et Rogers (2011) traitent de l'utilité des théories du changement (théories de programme) pour le suivi et lévaluation et proposent plusieurs exemples. On trouve aussi de bons exemples d'emploi des théories du changement, en particulier au stade de la planification et de la conception, dans Johnson, Guedenet et Saltzman (2014).

L'utilisation des théories du changement a été analysée par James (2011), Vogel (2012b), et Stein et Valters (2012). Tous observent qu'au-delà d'un consensus très général sur ce qu'est une théorie de changement - principalement sur les modèles décrivant comment l'intervention est supposée fonctionner - on observe 
une prolifération d'interprétations différentes sur ce qu'une théorie du changement comprend en pratique, sur la façon d'en élaborer une et de la décrire.

Dans le présent article, je propose un modèle pour l'élaboration de théories du changement qui soient utiles, pour les interventions simples ou complexes. Premièrement, je résume les principes généraux d'une théorie de changement, puis jaborde la question de la causalité. Ensuite, je présente lélaboration de modèles pour des interventions plus complexes multifacettes et je discute des théories du changement imbriquées. Je présenterai trois modèles possibles pour élaborer une théorie du changement, discuterai de la simplification des modèles et proposerai quelques observations sur la construction de théories du changement. Finalement, je résumerai les raisons qui expliquent en quoi ces modèles sont utiles.

\section{THÉORIES DU CHANGEMENT ET CHAÎNE D'EFFETS}

Commençons par définir quelques termes clés. Le terme résultats englobe les effets proximaux, les effets intermédiaires ainsi que les impacts, les impacts étant les derniers résultats avant le bien-être. J’emploie le terme intervention pour décrire des activités précises mises en œuvre afin de produire une différence souhaitable au niveau des effets. Le terme intervention est employé autant pour les politiques, les programmes et les projets.

Pour comprendre si une intervention fonctionne et comment elle fonctionne, nous devons comprendre comment les activités de l'intervention sont censées produire les résultats escomptés, c'est-à-dire : a) le cheminement causal entre les activités et les résultats depuis les effets les plus proximaux jusqu'aux impacts; b) les hypothèses causales qui indiquent pourquoi et dans quelles conditions les liens causaux sont supposés fonctionner. De nombreux termes sont utilisés pour désigner ces liens causaux : chaîne des résultats, modèle logique ${ }^{1}$ et chaînes d'effets. J'emploierai le terme " chaîne d'effets ».

Une chaîne d'effets désigne les liens de causalité entre les diverses étapes depuis les activités jusqu'aux impacts. Une théorie $d u$ changement ajoute à la chaîne d'effets les hypothèses causales qui sous-tendent chacun de ses liens, c'est-à-dire qu'elle explicite ce qui doit se produire pour que les liens de causalité se concrétisent. Patton (2008, p. 336) fait la même distinction entre les modèles logiques et les théories du changement : "Le fait de préciser les mécanismes de causalité transforme un modèle logique en une théorie du changement. » Chen (2015), au chapitre 3, fait une distinction similaire. Les théories du changement modélisent la façon dont le changement est censé se produire (cas ex ante) ou s'est produit (cas ex post).

Il y a bien des façons de dépeindre les chaînes d'effets et les théories du changement, comme l'illustrent d'ailleurs Funnell et Rogers (2011). La figure 1 représente une théorie du changement générique de base qui s'est avérée utile dans plusieurs contextes. La séquence des cases de la figure est la chaîne d'effets (chaîne de résultats), dont il sera d'abord question. La figure 1 est une version améliorée du modèle de théorie du changement présenté dans Mayne $(2014)^{2}$. 


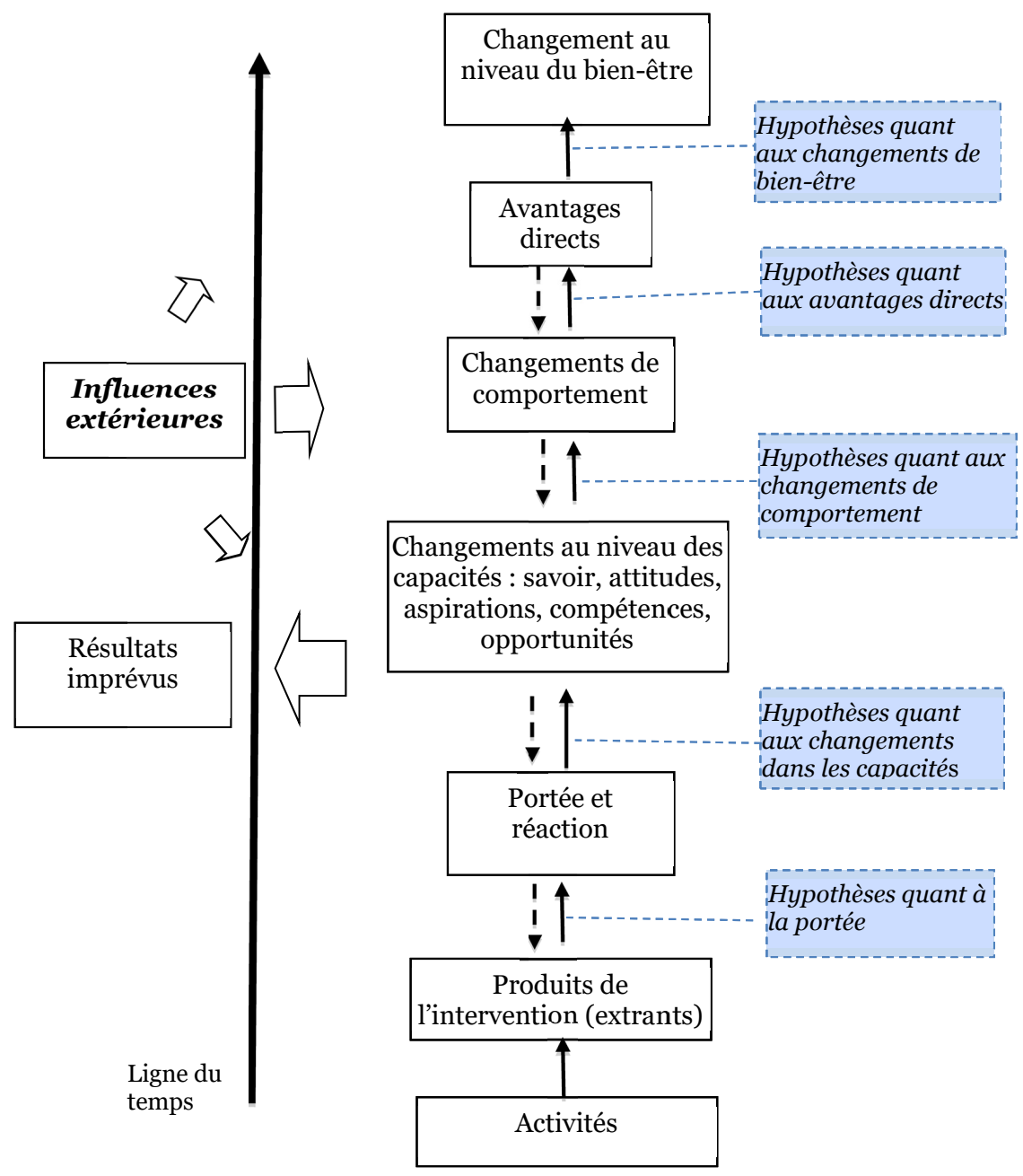

Figure 1. Théorie du changement générique et de base

\section{Les composantes d'une chaîne d'effets}

Les bénéficiaires sont les groupes cibles pour lesquels l'intervention vise à améliorer le bien-être. Ces groupes sont peut-être segmentés selon le niveau de revenu, le sexe, l'origine ethnique ou la région géographique. Prenons par exemple une intervention qui vise à améliorer la nutrition des enfants; les bénéficiaires visés sont les enfants. À noter qu'un groupe cible peut comprendre des organisations.

Dans le modèle de la théorie du changement, les activités et les résultats (des résultats proximaux depuis les produits jusquaux impacts) sont représentés dans les cases : 
- Les activités sont les actions entreprises par les personnes qui effectuent l'intervention.

- Les produits de l'intervention, biens et services, sont les extrants qui résultent des activités. Dans notre exemple de la nutrition, il pourrait s'agir du matériel éducatif sur les bienfaits d'une saine alimentation. Dans cet article, le terme produit désigne ces biens et services directs.

- La portée et la réaction sont, respectivement, les groupes cibles qui sont censés recevoir les biens et services de l'intervention et leur réaction initiale. Dans l'exemple sur la nutrition, la portée comprendrait le groupe des mères d’enfants d'une région géographique donnée. Il est important d'inclure la portée dans les chaînes d'effets. Comme l'ont déjà soutenu certains auteurs : «L'absence de réflexion explicite sur la portée dans les modèles logiques peut entraîner des problèmes tels qu'une compréhension étroite ou restreinte des chaînes d'effets, le favoritisme envers les initiatives "étroites et efficientes" au détriment des initiatives "larges et mobilisatrices”, ainsi qu'une réflexion biaisée qui écarte les considérations sur l'équité. » (Montague et Porteous, 2013, p. 177) J’ai discuté de l'utilité d'inclure la portée dans Mayne (2014).

- Les changements dans les capacités sont les changements liés aux savoirs de ceux qui ont reçu ou utilisé les biens et services de l'intervention, à leurs attitudes, compétences, aspirations et aux opportunités. Comme nous le verrons plus loin, tous ces changements sont nécessaires pour qu'une nouvelle action ${ }^{3}$ soit entreprise.

- Les changements de comportement sont les changements qui se produisent dans les pratiques réelles, lorsque les membres du groupe cible à rejoindre agissent différemment ou utilisent les produits de l'intervention. Dans notre exemple, il pourrait s'agir des changements dans les pratiques alimentaires des mères qui résultent de l'amélioration du savoir découlant de la formation. Il y a généralement une rétroaction entre la capacité et les changements de comportement (comme dans l'acquisition de nouveaux savoirs et de nouvelles compétences par l'action).

- Les avantages directs sont les améliorations de létat individuel des bénéficiaires. Il peut notamment s'agir d'une augmentation du revenu, d'une utilisation accrue des services de santé, de pratiques agricoles plus productives, de l'autonomisation des bénéficiaires ou, dans notre exemple, de la consommation d'aliments plus sains par les enfants.

- Les changements au niveau du bien-être ${ }^{4}$ se traduisent par une amélioration cumulative à long terme du bien-être général des bénéficiaires : l'amélioration de la santé, la réduction de la pauvreté, une sécurité alimentaire accrue. Dans notre exemple, l’amélioration du régime alimentaire contribuerait à bonifier la nutrition et l'état de santé des enfants.

À noter que le cheminement causal présenté à la figure 1 ne présente pas la séquence de résultats en effets proximaux, intermédiaires et finaux comme cest 
habituellement fait. Comme ces termes d'usage courant n'ont guère de signification intuitive, ils ne sont pas très éclairants pour établir la chaîne d'effets et leur utilisation ouvre souvent la porte à des débats stériles, à savoir si, par exemple, il sagit d'un résultat proximal ou intermédiaire. Et même si je pensais que le terme " extrants » était bien défini et généralement accepté, le récent guide du Groupe des Nations Unies pour le développement (2011) ajoute de la confusion en définissant les extrants comme des produits ou des changements de capacité. Je soutiens que la figure 1 est une représentation plus utile de la chaîne d’effets que le modèle courant.

Les influences extérieures sont les événements et conditions sans lien avec l'intervention qui pourraient affecter l'atteinte des résultats escomptés. Il peut s'agir d'autres interventions aux visées similaires ou encore de grandes tendances économiques ou sociales. Elles ne font pas partie inhérente de la théorie du changement relative à l'intervention. Dans notre exemple sur la nutrition, une baisse du prix des légumes pourrait aussi contribuer en partie à une augmentation de la consommation de légumes sans lien avec l'intervention éducative. Lenrichissement d'aliments industriels comme le sucre ou la farine pourrait également contribuer à expliquer une amélioration de la consommation de micronutriments.

La figure 1 indique les effets imprévus, soit les effets non anticipés positifs ou (plus souvent) négatifs qui résultent des activités de l'intervention. Si ces effets sont probables, il faut les représenter. Il importe de rechercher activement les effets imprévus ex post. Notons aussi que malgré son apparence linéaire, la figure 1 prévoit explicitement une non-linéarité au moyen de la rétroaction entre les divers stades. La figure 1 illustre aussi une ligne du temps indiquant à quel moment on peut s'attendre à ce que les changements escomptés se produisent. Une ligne du temps, même avec des dates approximatives, est un ajout utile à la chaîne d'effets.

\section{De la chaîne d'effets à la théorie du changement}

La première étape de l'élaboration d'une théorie du changement consiste à développer la chaîne d'effets. Mais une chaîne d'effets ou un modèle logique n'est pas une théorie du changement. C'est en ajoutant les hypothèses aux liens de causalité indiqués dans la chaîne des effets que nous obtenons une théorie du changement. Ces hypothèses illustrées par des cases pointillées dans la figure 1 indiquent les principaux événements et conditions qui doivent se produire pour que chaque lien de la chaîne des effets fonctionne comme prévu. Qu'est-ce qui est nécessaire au fonctionnement du lien de causalité? Quels facteurs sont essentiels à ces processus de causalité? Pour des raisons pratiques, il nous suffit de considérer les principales hypothèses, celles qui ressortent du lot pour une raison ou une autre, qui sont remarquables et qui sont pertinentes pour la situation. D'autres, comme le fait que le soleil se lève tous les matins ou qu'une révolution ne s'est pas produite, ne sont pas pertinentes, bien qu'ex post, une révolution expliquerait facilement pourquoi l'intervention n'a pas fonctionné! L'articulation d'hypothèses sur les liens de causalité implique un savant mélange d'évidences scientifiques, d'expérience des parties prenantes et de théories provenant des sciences sociales. Par exemple, 
dans le cas de l'alimentation des enfants, une hypothèse serait que le mari et la belle-mère approuvent les choix de la mère quant à l'alimentation des enfants et qu'ils la laissent prendre les décisions à ce chapitre.

Ces hypothèses sur les liens de causalité couvrent tous les risques associés à chaque lien de causalité; chacune des hypothèses est un risque pour la concrétisation de la théorie du changement ${ }^{5}$. Dans l'exemple de la nutrition, les risques relatifs à la disponibilité d'aliments nutritifs et à la modicité de leur prix seraient pris en compte dans l'hypothèse que des aliments nutritifs sont disponibles à prix modique.

- Hypothèses quant à la portée : Ces hypothèses sont les événements et conditions qui doivent être réunis pour que les produits de l'intervention (extrants) rejoignent les groupes cibles et soient reçus positivement par eux. Par exemple, on posera l'hypothèse que les produits de l'intervention rejoindront effectivement l'auditoire cible et que celui-ci les considérera comme acceptables et dignes d'intérêt. Ici, un des principaux risques est que le groupe cible ne soit pas le «bon " groupe, comme dans une intervention en nutrition infantile qui vise des mères alors qu'en fait, ce ne sont pas elles qui décident qui mangera quoi; il s'agit aussi de rejoindre la totalité du groupe ciblé et non, par exemple, uniquement les personnes qui sont volontaires pour participer.

- Hypothèses quant aux changements dans les capacités: Ces hypothèses sont les événements qui doivent se produire et les conditions qui doivent changer pour que les produits (extrants) qui atteignent les populations cibles induisent des changements au niveau de leurs savoirs, leurs attitudes, leurs compétences, leurs aspirations et de leurs opportunités - bref, leur capacité de faire les choses autrement. Par exemple, il pourrait s'agir que les produits soient compris, réalistes, culturellement acceptables, considérés comme utiles, cohérents avec les compétences et valeurs préalables de la population cible, considérés comme pertinents par le groupe visé.

- Hypothèses quant aux changements de comportement: Ces hypothèses sont les événements et conditions à réunir pour que les changements de capacité des groupes cibles se traduisent par une évolution réelle de leurs pratiques. On pourrait y inclure, entre autres, la capacité financière de réaliser les changements de pratique, l'acceptation par autrui (les pairs, les dirigeants sociaux, culturels et religieux, la famille) nécessaire à la réalisation des changements, la démonstration de l'utilité des changements de pratique, des politiques ou un cadre naturel favorables à l'adoption des pratiques, et l'accès aux biens et fournitures nécessaires.

- Hypothèses quant aux avantages directs : Ces hypothèses sont les événements et conditions nécessaires pour que les changements de pratique procurent un avantage direct sur les conditions des bénéficiaires ciblés. On pourrait notamment poser l'hypothèse qu'un changement de pratique 
entraîne une augmentation nette du revenu, une utilisation régulière des services de santé ou une participation à la prise de décision. Dans le cas de la nutrition, on peut poser l'hypothèse que le seul changement réalisé dans le régime alimentaire est celui que recommande le programme de formation. Par contre, si les pratiques améliorées (p. ex. consommer davantage de légumes) sont intégrées, mais que d’autres apports nutritifs sont réduits, lavantage attendu pourrait ne pas se concrétiser.

- Hypothèses quant aux changements sur le bien-être: Ces hypothèses sont les événements et conditions préalables nécessaires pour que les avantages directs entrainent des changements au niveau bien-être des bénéficiaires. Par exemple, si les enfants consomment des aliments plus sains et ont accès à des soins de santé de base et à de meilleures conditions d'hygiène, leur nutrition et leur état de santé s’amélioreront. Si, à la suite de l'intervention, les femmes commencent à jouer un rôle accru dans les décisions de consommation et que l'intervention est considérée comme réussie, ce changement pourrait contribuer à une évolution des normes de genre propre à renforcer le pouvoir d'agir des femmes.

On notera qu'une hypothèse sur le lien de causalité n’est pas une description du lien de causalité. La description d'un lien de causalité (ces liens sont représentés par des flèches continues dans la figure 1) serait, par exemple, que l'évolution des savoirs, des compétences et ainsi de suite (la capacité) se traduiront, en pratique, par les changements attendus de comportement. Les hypothèses sur les liens de causalité expliquent comment et pourquoi le lien de causalité fonctionne.

Il peut être très difficile de susciter des changements de comportement; bon nombre de chercheurs se sont penchés sur ce problème. Darnton (2008) recense une grande partie des écrits sur ce sujet. Le modèle des besoins, opportunités et compétences (needs, opportunities, and abilities - NOA) présenté dans Gatersleben et Vlek (1998) en est un exemple représentatif. Ce modèle postule que le changement de comportement est suscité par la motivation et par le contrôle du comportement (agence). De son côté, la motivation résulte des besoins et des opportunités, et l'agence découle des opportunités et des compétences. Tous ces éléments sont pris en compte dans la théorie du changement générique (savoirs, compétences, aspirations, attitudes et opportunités) en des termes différents, bien que les liens de causalité y soient moins détaillés. Mais la recherche suggère que chacune de ces composantes joue un rôle dans la relation causale, certaines étant directement visées par l'intervention, d’autres étant considérées comme des éléments pertinents pour comprendre les changements de comportement. Dans l'exemple de la nutrition, on peut supposer sans risquer de se tromper que les mères veulent améliorer la santé de leurs enfants (motivation) et que l'intervention vise à leur en donner la possibilité et la capacité.

Il peut être utile de distinguer deux types de changements de capacité et de comportement. Les premiers sont incrémentaux; ce sont des changements marginaux à létat actuel, par exemple l’apprentissage de techniques et de compétences 
nouvelles ou l'adoption de nouvelles pratiques. Ces changements sont plus faciles à créer que des changements fondamentaux, tels que le fait de penser les problèmes différemment ou de modifier ses pratiques actuelles. Dans notre exemple, si l'intervention vise l'acquisition de nouveaux produits alimentaires pour les enfants, il s'agit d'une pratique ajoutée et d'un objectif relativement simple. En revanche, si le changement de pratique recherché est une redistribution des aliments entre les membres du ménage, on s'attaque au mode actuel de distribution des aliments, ce qui soulève des enjeux de pouvoir. Ce changement fondamental sera vraisemblablement beaucoup plus difficile à implanter, de sorte que les hypothèses sur les liens de causalité qui y sont associées doivent être particulièrement robustes et ambitieuses.

Jusqu'ici, notre discussion est plutôt déterministe (par exemple, une hypothèse est nécessaire ou elle ne l'est pas). Mais nous pourrions aussi chercher à refléter la nature probabiliste de la causalité. Mahoney (2008, p. 421) avance qu' " un traitement est une cause lorsque sa présence augmente la probabilité qu'un résultat se produise dans n'importe quel cas ». Il présente les notions utiles de causes nécessaires en termes probabilistes (probabilistically necessary causes), soit des «facteurs qui doivent généralement ou presque toujours être présents pour que le résultat se produise ", et de causes suffisantes en termes probabilistes (probabilistically sufficient causes), au sens d'une " cause qui, la plupart du temps, produit l'effet d'elle-même» (p. 425-426). Pour bien des interventions visées par une évaluation, il s'agit d'interprétations réalistes des notions de nécessité et de suffisance.

Ainsi, on peut envisager les hypothèses sur les liens de causalité comme des hypothèses, des événements et des conditions probablement nécessaires qui, presque toujours, doivent se produire pour que le lien de causalité fonctionne.

Énoncer des hypothèses associées à une théorie du changement peut être difficile, en raison de la diversité des types d'hypothèses associés à une intervention. En particulier, outre les hypothèses sur les liens de causalité dont il a été question plus haut, il existe aussi des hypothèses de rationalité, qui décrivent l'hypothèse ou prémisse sous-jacente servant de fondement à l'intervention. Par exemple, on présume que le fait d'informer les adultes d'un ménage des bienfaits d'une bonne nutrition pour leurs enfants entraînera un changement de comportement de leur part et une amélioration du régime alimentaire des enfants. On présuppose que la justification d'une intervention repose généralement sur des données probantes et sur l'expérience antérieure.

La figure 2 illustre la théorie du changement associée à l'exemple de la nutrition ${ }^{6}$. Cet exemple n'est pas fondé sur un cas réel, mais White (2009) analyse une intervention très semblable menée au Bangladesh.

J'avance que, dans la plupart des interventions, chacune des composantes de la théorie du changement générique - les activités, les produits, les changements dans les capacités, les changements de comportement, les avantages directs, les changements au niveau du bien-être, de même que les hypothèses sur les liens de causalité associées - peuvent et doivent être déterminées et faire l'objet d'une 


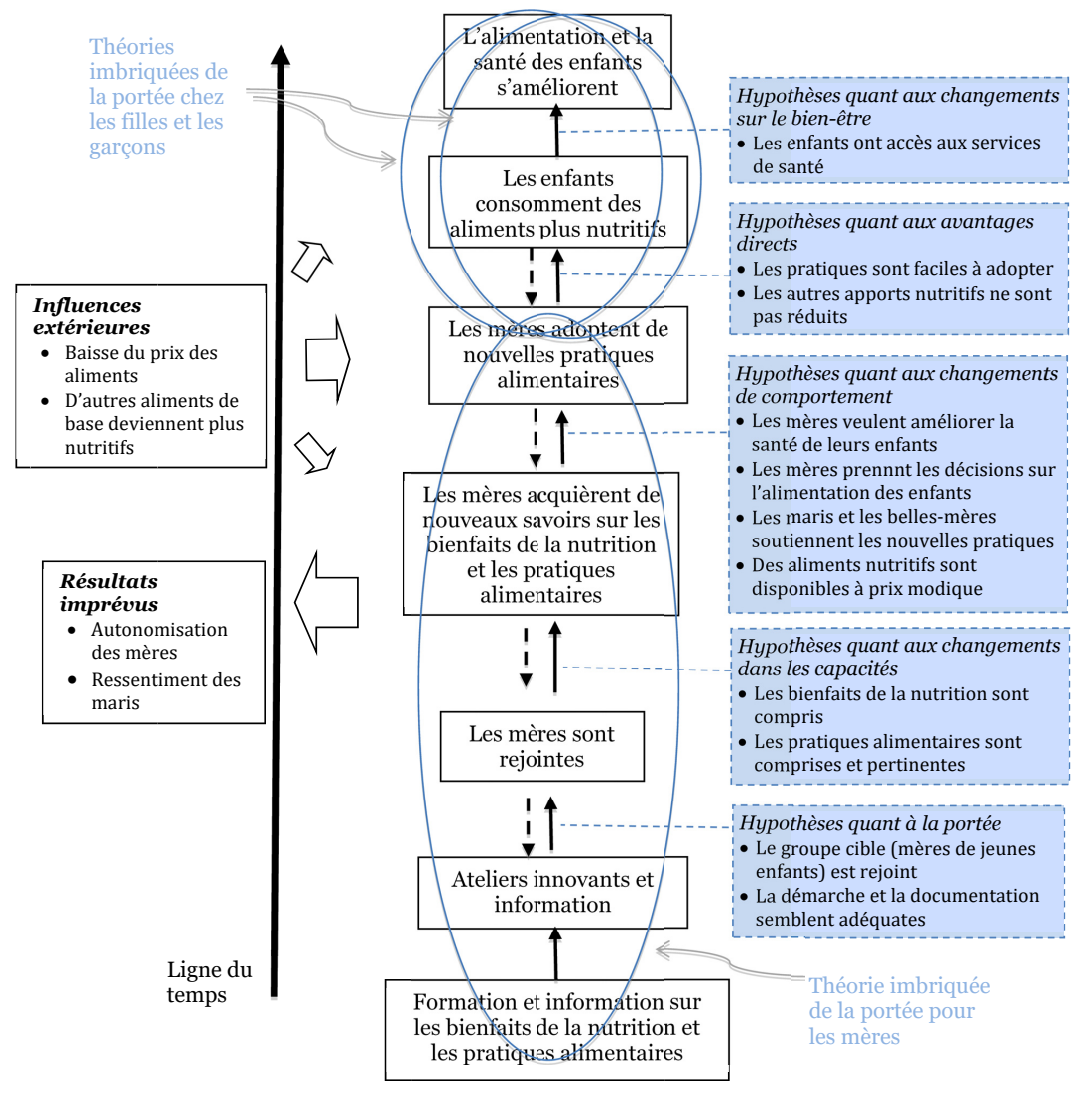

Figure 2. Théorie du changement d'une intervention en nutrition

réflexion lors de lélaboration de chaînes des effets et de théories du changement. La structure du modèle nous force à considérer la façon dont on s’attend à ce que les résultats escomptés surviennent : Quel est le processus de causalité à l’œuvre? Que faut-il pour qu'il se concrétise? Le modèle est un cadre pour l'analyse du fonctionnement d'une intervention.

\section{THÉORIES DU CHANGEMENT ET FAISCEAUX DE CAUSALITÉ}

Les théories du changement illustrent comment et pourquoi on sattend à ce qu'une intervention contribue à l'atteinte des effets escomptés. Toutefois, pour qu'ils se produisent, il faut plus que la simple réalisation des activités; il faut aussi que les hypothèses sur la causalité se manifestent. Les activités sont rarement la seule cause d'un effet. La théorie du changement décrit un faisceau de causalité constitué d'activités et d'hypothèses qui, ensemble, sont censées contribuer à 
l'obtention des résultats escomptés (condition de suffisance). Cartwright et Hardie (2012) appellent ces hypothèses des facteurs de soutien (support factors), c'est-àdire des événements et conditions essentiels pour contribuer à l'obtention des effets. On s'attend aussi à ce que les activités constituent une portion essentielle de ce faisceau de causalité (causal package) - condition de nécessité. Autrement dit, sans les activités, la manifestation des hypothèses sur les liens de causalité ne suffirait pas à produire l'effet escompté. On dit alors que les activités sont une cause contributive des résultats. Selon ces termes, une théorie du changement est un modèle de contribution causale de l'intervention; c'est un modèle qui indique l'ensemble des liens de causalité et leurs contributions à l'obtention des résultats. Mayne (2012) aborde la question des causes contributives et les faisceaux de causalité dans le contexte des théories de la causalité et plus particulièrement des causes INNNS 7 .

La théorie du changement est un modèle de la contribution aux effets attendus et non la cause de cet effet, car d'autres facteurs extérieurs peuvent aussi contribuer à l'obtention des résultats escomptés, comme l'indique la case «Influences extérieures ». La théorie du changement n'est un modèle de causalité que si aucune influence extérieure n'entre en jeu. Comme une intervention, une influence extérieure peut fonctionner isolément, mais elle peut aussi faire partie d'un autre faisceau de causalité qui pourrait inclure certains des facteurs de soutien du faisceau de causalité de l'intervention. Les influences extérieures peuvent avoir des effets positifs ou négatifs sur le niveau des résultats obtenus. Tout dépendant des forces de l'intervention, les influences extérieures peuvent expliquer tout, ou en partie, les résultats observés. Les effets négatifs importants, c'est-à-dire les risques susceptibles de saper la théorie du changement de l'intervention, sont inclus dans les hypothèses sur les liens de causalité.

En termes probabilistes, l'expression vraisemblablement suffisante peut servir à décrire le niveau de suffisance du faisceau de causalité de l'intervention, ce qui signifie que le faisceau de causalité a probablement contribué à lobtention du résultat observé. Démontrer que l'intervention est une cause contributive équivaut à démontrer que son faisceau de causalité est vraisemblablement suffisant et que l'intervention, en soi, constitue un élément vraisemblablement nécessaire de ce faisceau.

Dans l'analyse des théories du changement, il est utile de distinguer le ex ante du ex post. Ex ante, il est nécessaire de parler des causes probabilistes et de la probable suffisance. Ex ante, on dispose d'une théorie du changement préliminaire ou postulée qui dit que si l'intervention est implantée telle que conçue et que si les hypothèses associées à la théorie du changement tiennent, alors la contribution anticipée aux résultats se concrétisera. Ce modèle assume la réalité et rend compte de la complexité de l'intervention. Il constitue une prédiction de l'efficacité de l'intervention.

Ex post, nous vérifions que la théorie du changement s'est effectivement réalisée, en utilisant les données probantes sur les résultats et sur la manifestation des hypothèses. Lorsque l'analyste conclut à une relation de causalité, il le fait en sachant quels facteurs étaient à l'œuvre et si d'autres éléments ont interféré. Si 
l'analyste conclut que le faisceau de causalité était vraisemblablement suffisant, il reconnaît qu'un élément a peut-être échappé à l’analyse, mais qu'une personne raisonnable conclurait que le faisceau de causalité était effectivement suffisant. Ex post, nous vérifions l'hypothèse causale formulée ex ante. Comme il a été noté, ex post, nous sommes probablement en mesure de déterminer si d'autres influences extérieures sont entrées en jeu. Dans le cas contraire, nous pourrons dire que le faisceau de causalité de l'intervention a bel et bien causé le résultat observé, audelà de la simple contribution.

L'intervention en soi est un des nombreux facteurs de causalité du faisceau de causalité nécessaire pour susciter un changement. En ce sens, tous sont égaux. Pourtant, notre intérêt se porte sur l'intervention comme instrument de changement, principalement sur les activités implantées dans le but d’obtenir un changement ou de le maintenir. Nous pouvons nous interroger, ex post, sur le rôle de l'intervention dans la survenue des changements. Nous pouvons nous attendre, au minimum, à ce que l'intervention serve de déclencheur pour démarrer la chaîne de causalité. Dans un tel cas, on pourra dire que l'intervention est une cause contributive principale. Parfois, l'intervention peut aussi être vue comme jouant un rôle complémentaire qui en s'ajoutant à d'autres éléments d'un processus déjà en cours, bonifie un changement déjà amorcé afin d’optimiser les résultats ou d'accélérer leur atteinte (Mayne, 2008).

\section{LES INTERVENTIONS MULTIFACETTES SUFFISANTES}

Malgré tout ce quelle contient, jai décrit la figure 1 comme une théorie du changement générique " de base ». C'est qu’elle ne présente qu'un seul acteur qui entreprend des activités; ce modèle peut suffire pour bon nombre d'interventions simples, mais pas pour la plupart des interventions complexes. Pour entraîner des changements, une intervention doit faire appel et sassocier à divers autres intermédiaires - partenaires de la prestation de services, administration publique, secteur privé, ONG - et influencer leur comportement. La théorie du changement illustrée à la figure 1 fait ressortir un éventail potentiellement large d'hypothèses de liens de causalité, qui doivent se concrétiser pour produire les avantages directs et les changements sur le bien-être. S'en remettre au hasard nest peut-être pas une option, donc l'intervention doit s'associer à des intermédiaires compétents, notamment des partenaires de la prestation de services, pour veiller à ce que les actions qu'ils entreprennent assurent (ou contribuent à assurer) la concrétisation des nombreuses hypothèses de liens de causalité. Ces activités de soutien menées par les acteurs de l'intervention s'ajoutent aux activités principales ou de base de l'intervention. On peut donc distinguer l'intervention de base et l'intervention globale. Dans une intervention sur la recherche en agriculture, l'intervention de base correspond aux activités de recherche, tandis que l'intervention globale regroupe ces activités plus celles qui soutiennent l'engagement et qui sont menées pour favoriser l'utilisation des résultats de la recherche. Dans d'autres cas, il n’y 
a aucune activité de base ou principale : l'intervention s'associe à un éventail de partenaires pour offrir collectivement un ensemble d'activités suffisant.

Le plus souvent, ces hypothèses de liens de causalité couvrent une gamme d'événements ou de conditions qui créent un cadre supportant pour que les activités d'intervention contribuent au bien-être. Il en résulte une intervention globale multifacette, dont l'objectif est de rendre l'intervention suffisante : que l'ensemble des efforts d'intervention (de base), ses activités de mobilisation et les actions résultantes des intermédiaires influence effectivement les avantages et le bien-être. Autrement dit, l'ensemble des activités de mobilisation vise à assurer la concrétisation des hypothèses de liens de causalité, c'est-à-dire celle des facteurs de soutien.

Nous pouvons tout de même nous demander si l'intervention de base a été une cause contributive principale, autrement dit, si elle a contribué à déclencher le changement. Et dans les contextes de suffisance multifacettes, l'intervention implique la mobilisation de diverses actions de soutien afin de soutenir la réalisation du cheminement causal. Ainsi, il est important d'évaluer si l'intervention de base est une cause contributive de déclenchement et une cause contributive de soutien.

Une solide inférence causale au sujet d'une intervention multifacette suffisante serait que l'intervention a été une cause contributive principale des résultats pertinents observés :

L'intervention a été une composante nécessaire d'un faisceau de causalité qui, ensemble, ont été suffisants dans leur contribution au résultat observé. Autrement dit, l'intervention a fait une différence. En outre, l'intervention a joué un rôle clé : elle a déclenché et, par ses activités de soutien, appuyé la chaîne d’effets qui a contribué aux résultats observés.

La figure 3 illustre la théorie du changement générique dans cette intervention complexe, qu'on peut qualifier d'intervention multifacette ${ }^{8}$ suffisante.

Quand on construit une théorie du changement, il peut être utile de déterminer le degré de contrôle réel ou éventuel que l'intervention a sur les hypothèses de liens de causalité. On donne à chaque hypothèse une étiquette : [O], si l'intervention n'exerce aucune influence ou presque; [I], si l'intervention peut (devrait) exercer une influence directe ou indirecte; [C], si l'intervention devrait parvenir à exercer un contrôle direct. Cette stratégie aide à déterminer où des actions de soutien supplémentaires pourraient contribuer à la concrétisation des hypothèses et, partant, à une réduction au minimum des risques pour l'intervention, ce qui pourrait mener à une intervention multifacette.

À partir de l'exemple de la nutrition, le tableau 1 illustre le type d'analyse ex ante des liens de causalité que l'on peut entreprendre. Pour chacune des hypothèses de la figure 2, on évalue la mesure dans laquelle l'intervention pourrait investir des efforts pour renforcer la probabilité que l'hypothèse se réalise.

Dans l'exemple, il se peut que les maris ou les belles-mères soient peu susceptibles d'aider les mères à prendre des décisions sur la répartition des aliments. Donc, pour fonctionner, l'intervention nécessite une certaine forme d'éducation des maris et des belles-mères sur les avantages d'une alimentation nutritive pour 


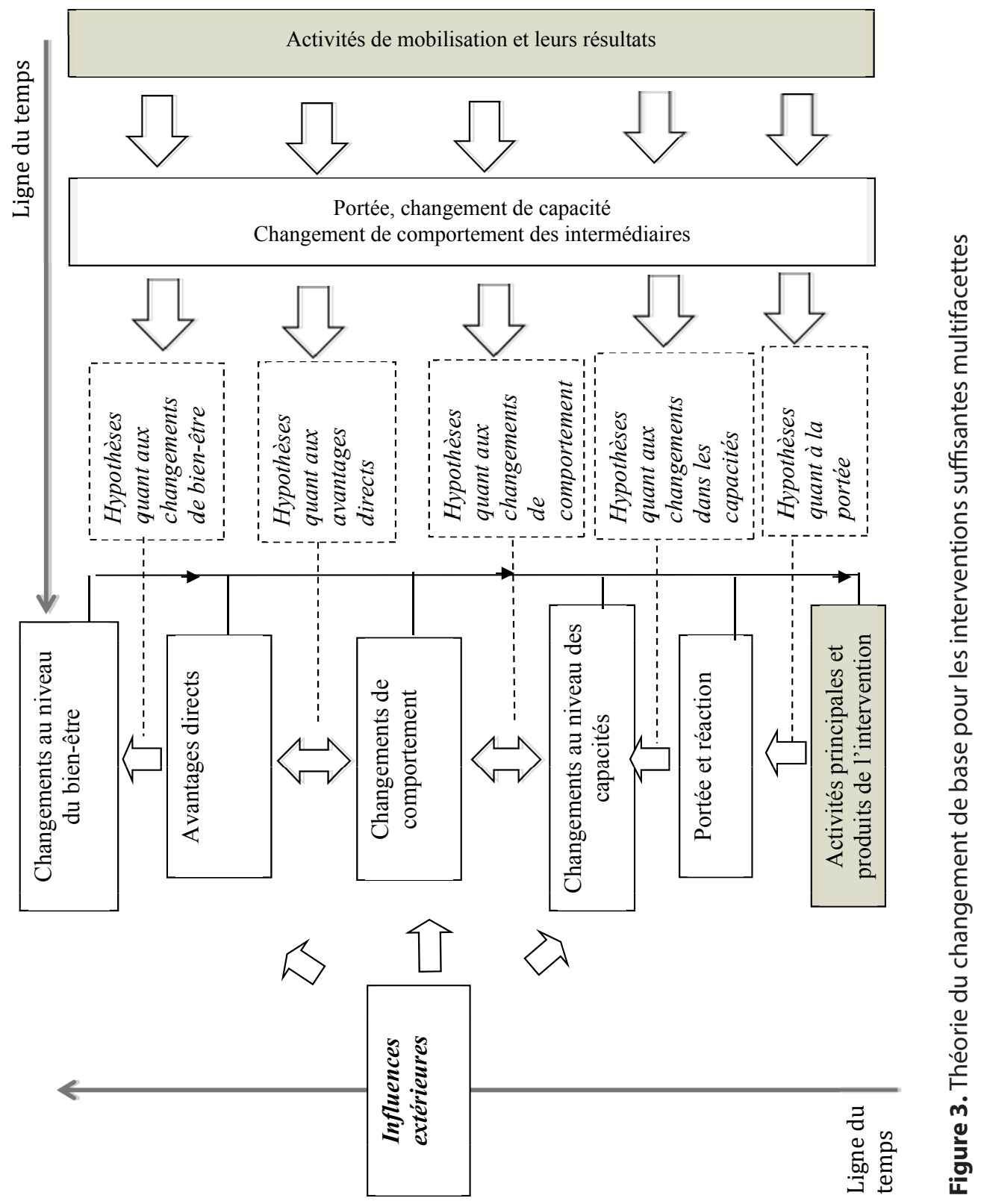


Tableau 1. Analyse des hypothèses de liens de causalité dans une intervention en nutrition

\section{Hypothèse de lien de} causalité
Degré de Action de soutien nécessaire en plus des contrôle activités de base

A1 Hypothèses quant à la portée

- Le groupe cible (mères de Moyen [I] L'intervention doit connaître sa populajeunes enfants) est rejoint. tion cible et savoir comment la rejoindre.

Action : Nécessite probablement des activités d'information.

- La démarche et la documenta- Élevé [C] Exige une bonne planification et une contion semblent adéquates. naissance du contexte particulier.

A2 Hypothèses quant aux changements dans les capacités

- Les bienfaits de la nutrition Élevé [C] Exige une bonne planification et une sont compris. connaissance du contexte particulier.

- Les pratiques alimentaires Élevé [C] Exige une bonne planification et une sont comprises et connaissance du contexte particulier. pertinentes.

\section{A3 Hypothèses quant aux changements de comportement}

- Les mères veulent améliorer la s.o. Peut être tenue pour acquise. santé de leurs enfants.

- Les mères prennent les Inconnu Nécessiterait une connaissance du décisions sur l'alimentation contexte particulier. des enfants.

- Les maris et les belles-mères Faible [I] Action : Nécessite une approche des maris soutiennent les nouvelles pratiques. et des belles-mères sur l'importance d'un régime nutritif pour les enfants.

- Des aliments nutritifs sont Élevé [C] Préalable de l'intervention. Si cette disponibles à prix modique. disponibilité est peu probable, un type d'intervention différent (des subventions, par exemple) sera nécessaire.

\section{A4 Hypothèses quant aux avantages directs}

\section{- Les pratiques sont faciles à} adopter.

\section{- Les autres apports nutritifs} ne sont pas réduits.

\author{
Moyen [I]
}

Action : Pourrait nécessiter un suivi pour vérifier si les pratiques sont faciles à adopter dans le contexte particulier.

Élevé? [C] Devrait faire partie de la formation : ne pas arrêter de consommer d'autres aliments nutritifs. Mais il existe un risque que les maris et les belles-mères des ménages défavorisés insistent sur une substitution. Action : Nécessite une approche des maris et des belles-mères.

\section{A5 Hypothèses quant aux changements sur le bien-être}

\section{- Les enfants ont accès aux ?? [O]} services de santé.
Sera probablement simplement tenue pour acquise. Si la santé est un problème majeur, cela pourrait remettre l'intervention en question. 


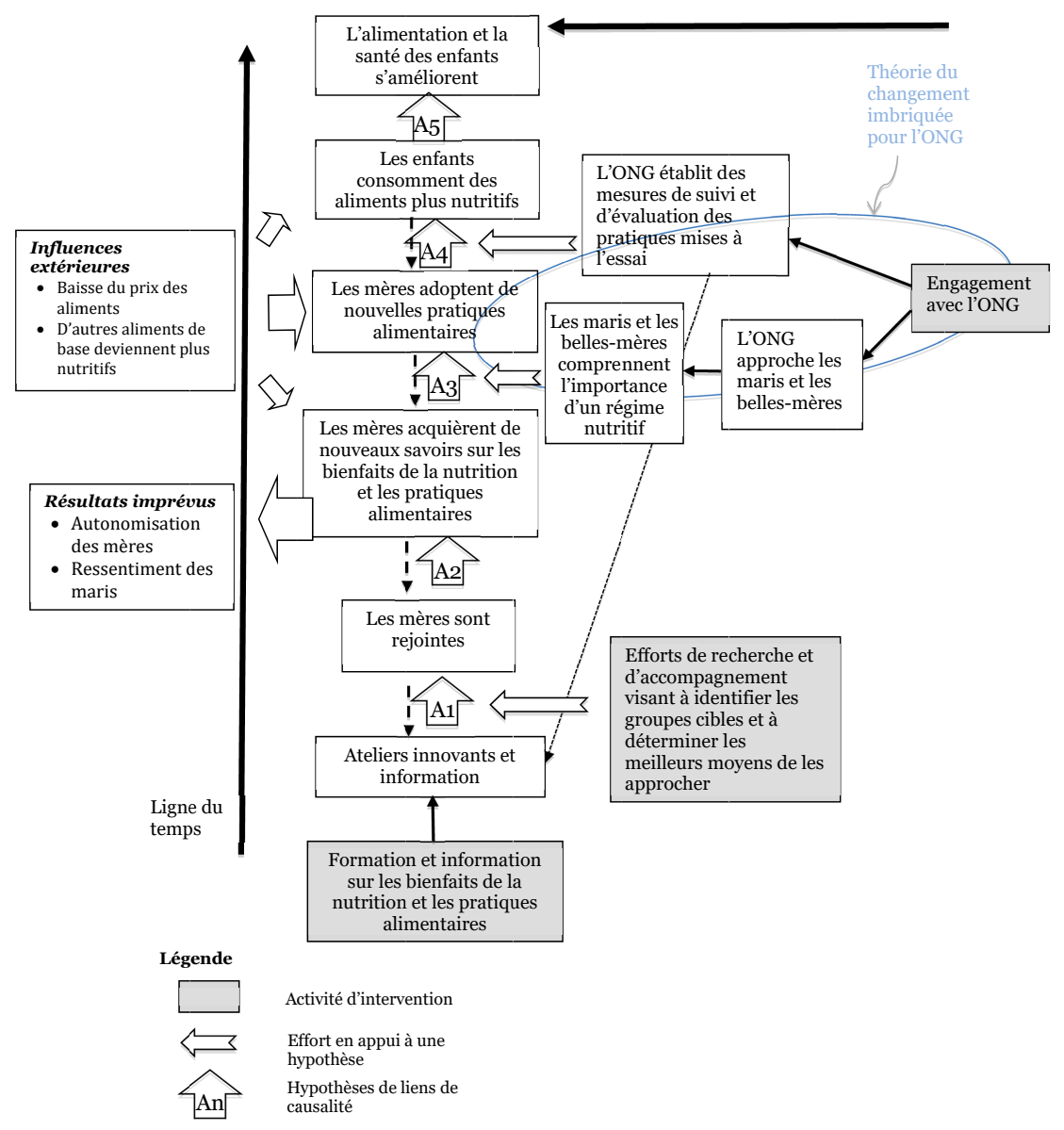

Figure 4. Intervention multifacette en nutrition

les enfants. L'organisme d'intervention devra peut-être amener d'autres parties prenantes qui ont davantage l'habitude de traiter des questions de culture et de genre, une ONG par exemple, à approcher les maris et les belles-mères afin d'influencer leur comportement. La figure 4 illustre l'intervention multifacette en nutrition qui en résulte.

Lanalyse ex ante des liens de causalité peut aussi servir à évaluer a priori dans quelle mesure des données empiriques soutiennent chaque lien d'une théorie du changement. Parfois, il existe des recherches et des résultats dévaluations antérieures qui permettent de documenter certains liens de causalité. Il arrive aussi que les informations existentes soient de faible qualité ou inexistantes. Dans ce dernier cas, il serait justifié d’entreprendre une nouvelle recherche afin de mieux confirmer le lien de causalité avant de mettre en œuvre l'initiative, ou encore d'assurer un suivi attentif des hypothèses rattachées à ces liens au cours de la mise 
en œuvre de l'intervention. Ce type d'analyse des liens de causalité est traité par Mayne et Johnson (2015) et par Johnson, Mayne, Grace et Wyatt (2015).

Bien sûr, l'analyse ex post des liens de causalité d'une théorie du changement est tout aussi intéressante. Déterminer dans quelle mesure un lien de causalité et ses hypothèses se sont réalisées et apprécier la crédibilité du cheminement causal est l'essence même de l'analyse de la contribution (Mayne, 2008; Mayne, 2012).

\section{LES INTERVENTIONS COMPLEXES}

Les figures 1 et 3 ciblent un seul groupe de bénéficiaires, les enfants dans l'exemple de la nutrition (figure 2). Toutefois, les interventions ont souvent plusieurs groupes cibles (les mères et les enfants, par exemple) ou plusieurs sous-groupes d'un groupe général (les garçons et les filles). Une intervention multifacette suffisante (figure 3) cible généralement plusieurs intermédiaires différents (administrations publiques, organismes, partenaires). Dans ce genre d'intervention à cibles multiples, une approche consiste à essayer d'élaborer une théorie du changement qui englobe toutes les activités menées sur les divers groupes cibles et les séquences de résultats qui s'ensuivent, de même que les liens entre les divers cheminements. Cependant, il risque de s'avérer très difficile d'élaborer et de représenter un tel modèle au-delà du simple survol (lequel a tout de même son utilité) et le modèle de théorie du changement complexe qui en résulte est souvent lourd et peu maniable, qu'il soit utilisé pour expliquer l'intervention ou pour concevoir l'évaluation.

\section{Les théories du changement imbriquées}

Il serait donc beaucoup plus utile délaborer, pour chacun des principaux groupes cibles, une sous-théorie du changement, appelée théorie du changement imbriquée ou théorie de la portée ${ }^{9}$, chaque théorie étant éventuellement en interaction avec les autres théories dans la chaîne de production des effets. La figure 2 illustre les théories de la portée imbriquées (représentées par des ovales) pour les mères et pour les filles et les garçons. Garçons et filles pouvant faire l'objet de traitements différents, une théorie de la portée distincte pour chacun de ces sous-groupes ferait ressortir ces différences.

La figure 4 illustre la théorie du changement imbriquée applicable à une ONG dans l'exemple de la nutrition, qui est présenté en détail à la figure 5. Les hypothèses incluses dans la théorie du changement pour l'ONG (figure 5) sont celles que l'ONG devrait être à même de contrôler ou d'influencer fortement. La figure 6 illustre les théories de la portée imbriquées pour l'intervention multifacette suffisante générique. Les théories du changement imbriquées sont un moyen de décomposer une théorie du changement complexe en composantes plus maniables et plus faciles à comprendre.

\section{L'analyse des liens de causalité}

Pour traiter une théorie du changement complexe, on peut aussi analyser séparément les principaux liens de causalité, par exemple le lien entre « les changements 


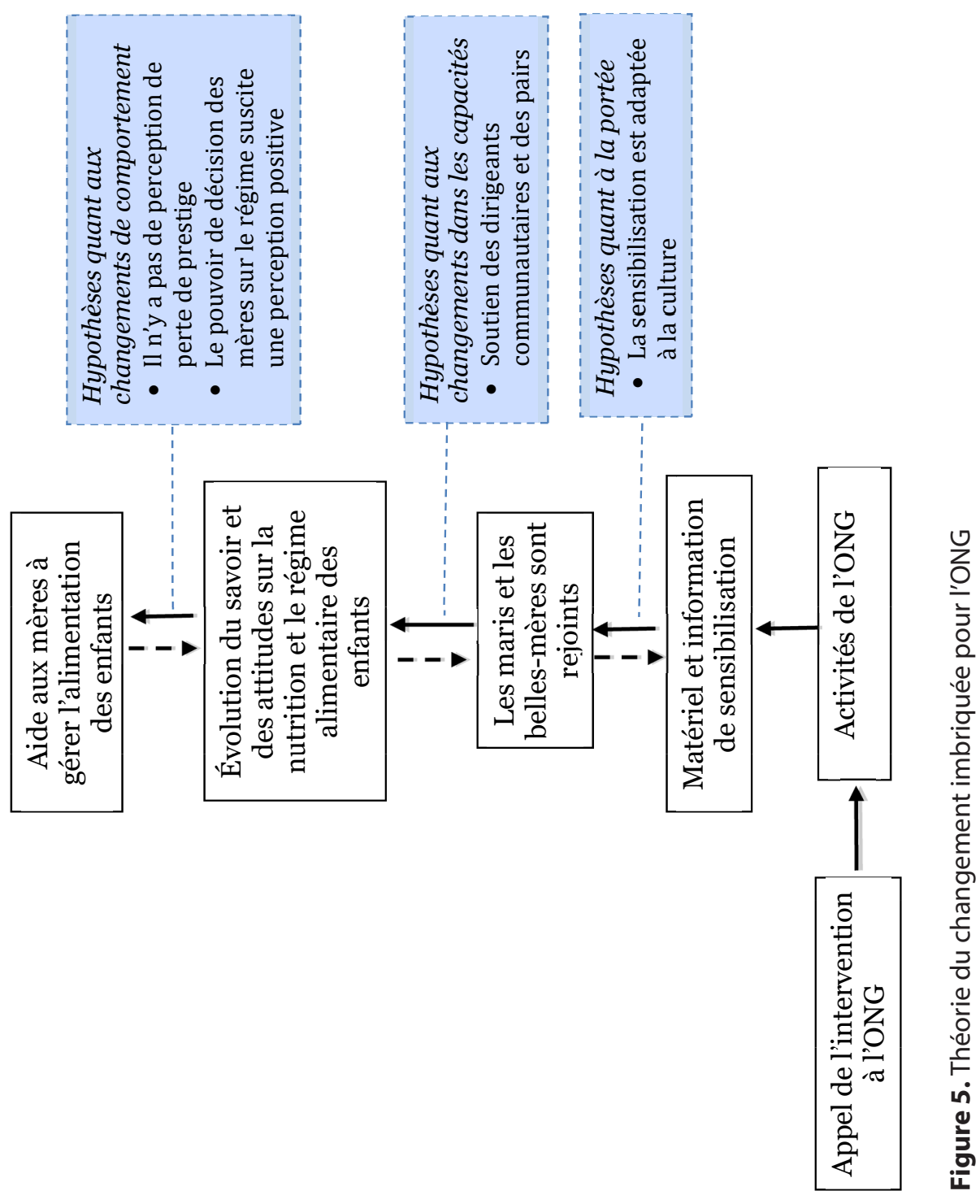



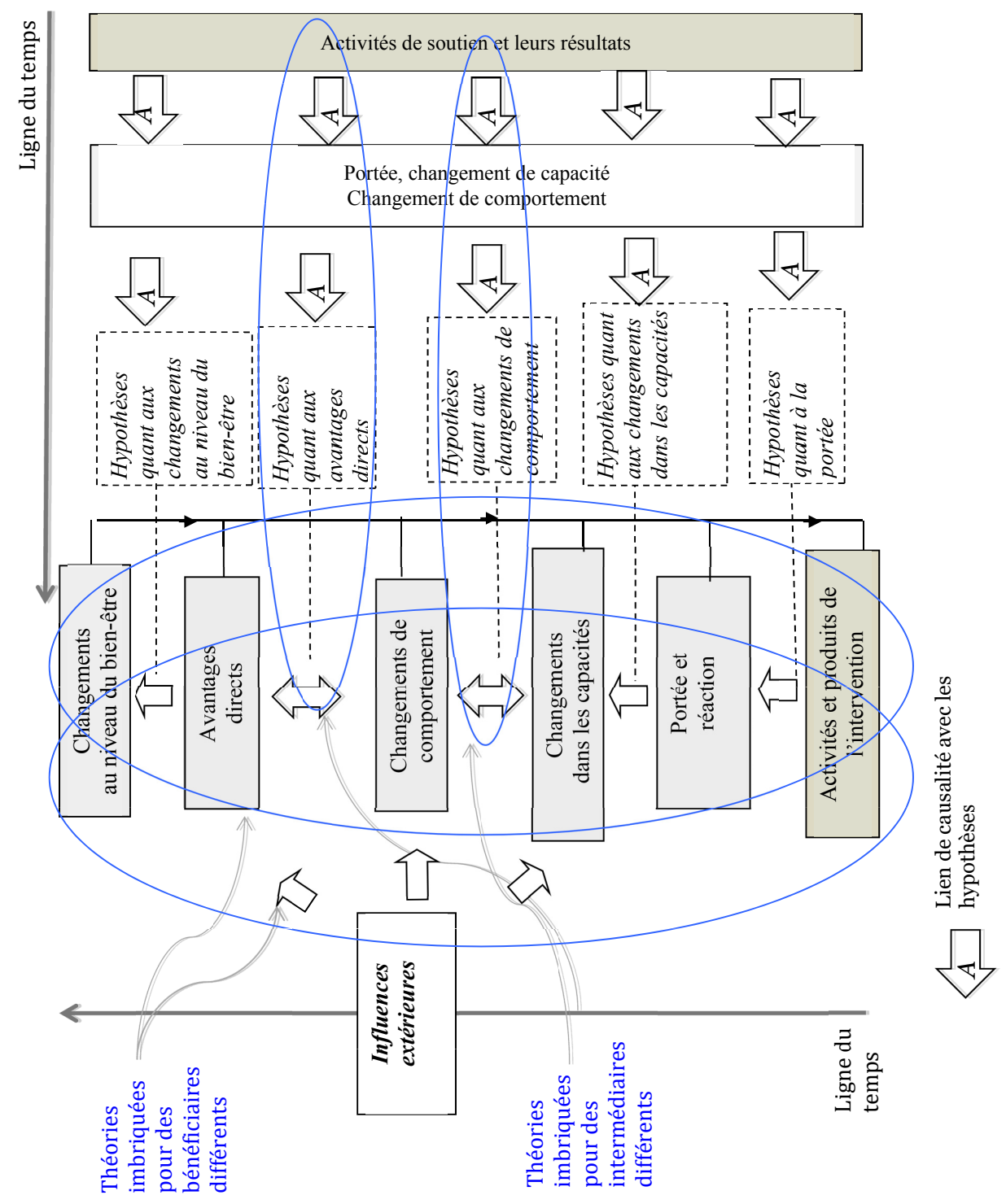

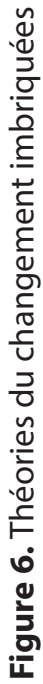


dans les capacités et les changements de comportement ». De plus, l'analyse des diverses hypothèses peut, par exemple, se faire dans un texte explicatif contenant des renvois aux recherches et évaluations antérieures qui appuient ces hypothèses, comme on l'a fait au tableau 1. Mayne et Johnson (2015) illustrent cette approche.

\section{L'incertitude et les résultats émergents}

Les interventions varient dans leur niveau de complexité, des interventions compliquées telles que les interventions multifacettes suffisantes décrites plus haut, jusquà des interventions réellement complexes qui présentent un grand niveau d'incertitude et des propriétés émergentes. Pour l'évaluation des interventions vraiment complexes, on suggère habituellement d'utiliser lévaluation pour favoriser les apprentissages et les changements incrémentaux sur une certaine période de temps (Ling, 2012; Mayne, 2011, p. 82-84; Rogers, 2011; Sanderson, 2000). Dans cette optique, il serait logique délaborer des théories du changement initiales, comme celles que nous avons présentées ici, puis de les réviser et les adapter en fonction des nouvelles connaissances qui pourraient émerger. Rogers (2008) et Ling (2012) traitent de l'utilisation des théories de programme et des théories du changement dans les contextes complexes.

\section{DIFFÉRENTES THÉORIES DU CHANGEMENT POUR DIFFÉRENTS OBJECTIFS}

Il y a des limites évidentes au niveau de détail qu’on peut inclure dans le modèle d'une théorie du changement, surtout dans le cas d'une vaste intervention multifacette. Nous avons besoin d'outils plus maniables, tant pour travailler le modèle quà des fins de communication. Il est donc utile de disposer d'au moins trois versions de chaque théorie du changement.

La première de ces versions, le texte explicatif, décrit en une ou deux phrases le fonctionnement prévu de l'intervention planifiée ou mise en ouvre. Cette version de la théorie du changement explique sans détour comment lintervention est censée fonctionner; on peut aussi y énoncer les hypothèses sous-jacentes à l'intervention. C'est cette description de base, ce " synopsis », cet énoncé qu'un gestionnaire (ou un politicien) donne quand on lui demande d'expliquer pourquoi, à son avis, l'intervention va fonctionner. Prenons par exemple la théorie simple applicable à une intervention ayant la forme d'une publicité télévisée contre le tabagisme : si on décrit les dangers du tabagisme à la télé, des fumeurs cesseront de fumer. Dans l'exemple de la nutrition des enfants, la version " texte explicatif » de la théorie du changement pourrait se lire comme suit : « Léducation et l'information des mères sur l'importance d'une alimentation nutritive pour leurs enfants les amèneront à modifier leur comportement et à chercher à améliorer le régime alimentaire de leurs enfants. » Ici, l'hypothèse sous-jacente sur laquelle repose l'intervention est qu'une meilleure information entrainera un changement de comportement.

Le texte explicatif de la théorie du changement joue un rôle important, car c'est ce message qui sera repris lorsque l'intervention sera présentée ou défendue 


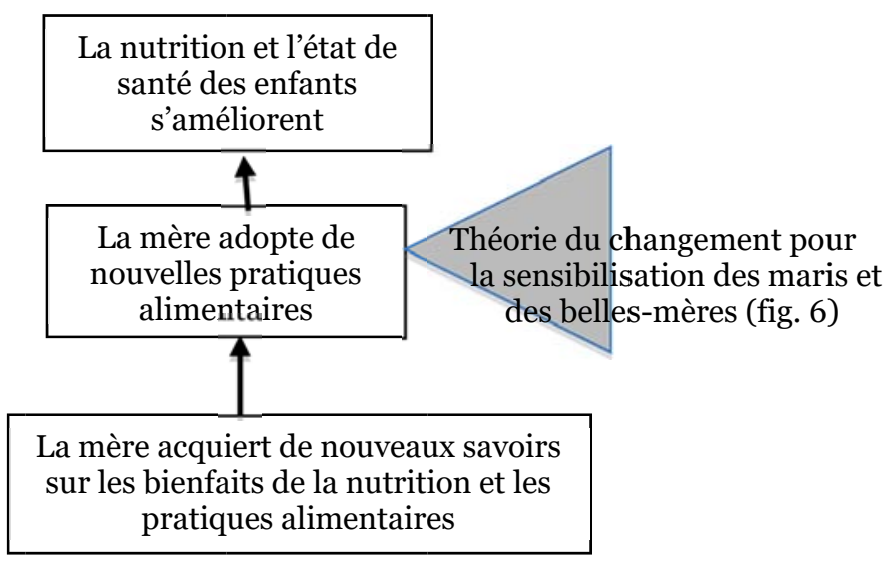

Figure 7. Survol d'une intervention en nutrition

sur la place publique. Ce message constitue, en quelque sorte, la version publique de la théorie du changement.

Une deuxième version de la théorie du changement, est un résumé de la théorie de changement, qui dresse globalement le portrait d'une intervention multifacette. Ce résumé peut être un schéma simplifié de la chaîne des effets indiquant les théories de changement imbriquées et les principales hypothèses. La figure 7 reprend l'exemple de la nutrition et de sa théorie du changement pour la sensibilisation des maris et des belles-mères représentée, ici, par un triangle.

La troisième version est plus détaillée. La théorie causale de changement est habituellement représentée sous forme de graphique, comme ceux des figures 1 à 5 , qui indiquent la chaîne des effets et les différents liens de causalité. Chacune de ces versions de la théorie du changement a son utilité et il est souvent avantageux d'avoir les trois versions à sa disposition.

Un autre moyen de simplifier le modèle d'une théorie du changement consiste à omettre les «boites » et à inclure l'essentiel de leur contenu dans les hypothèses sur les liens de causalité, ce qui équivaut à réorganiser le faisceau de causalité. Pour bon nombre d'interventions, le regroupement de tous les éléments de la chaîne des effets ou de la théorie du changement dans un seul diagramme rend le graphique trop complexe et le lecteur se perd entre les flèches et les cases. La figure 8 illustre une version "simplifiée » de la figure 1, où les cases « Portée » et « Changements dans les capacités » ont été éliminées. Il est souvent tentant d’agir ainsi, car ce sont les changements de comportement qui sont considérés comme les principaux résultats du cheminement d'impact.

Comme nous le voyons dans la figure 8, la chaîne des effets est représentée de façon synthétique. Il faudra toutefois se rappeler que les aspects de la portée et des changements dans les capacités ne sont pas explicitement indiqués. Dans ce cas, les hypothèses allant des activités et produits de l'intervention aux changements 


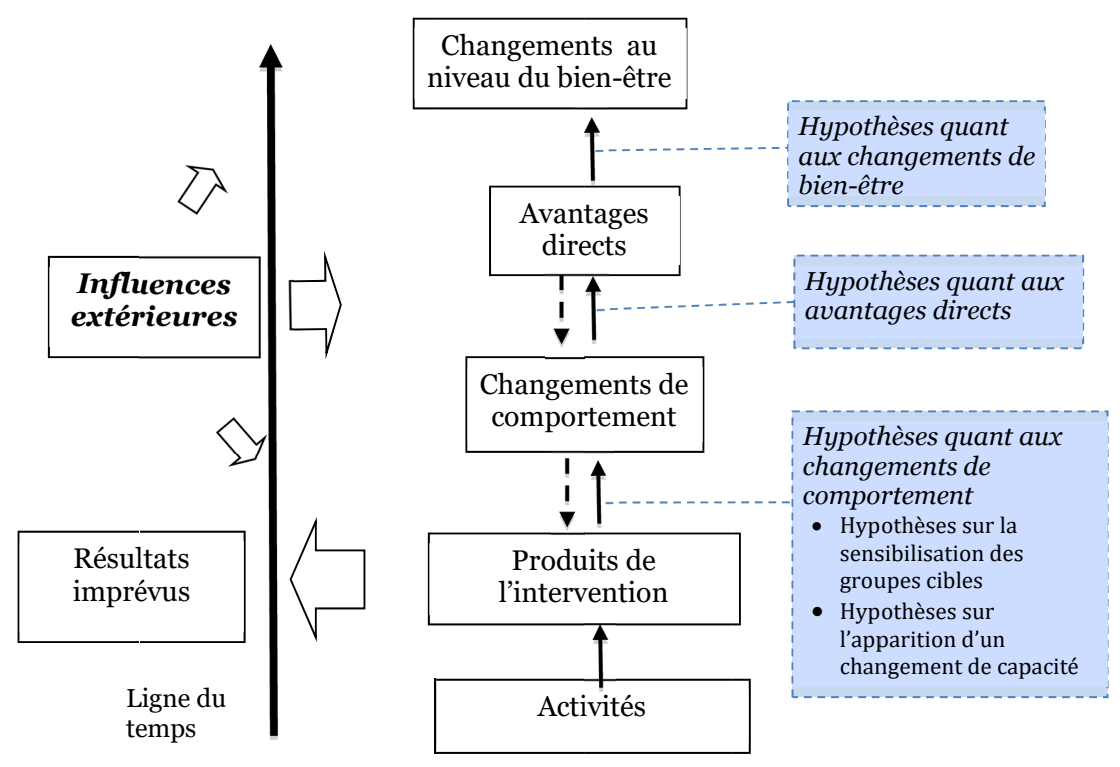

Figure 8. Version simplifiée d'une théorie du changement générique

de comportement devront inclure les hypothèses relatives à la portée et aux changements dans les capacités. Autrement dit, le faisceau de causalité associé à chaque lien demeure intact; il est simplement présenté différemment. Lomission de prendre en compte les enjeux associés à la portée et aux changements dans les capacités affaiblirait significativement la théorie du changement.

\section{LA CONSTRUCTION DES CHAÎNES D’EFFETS ET DES THÉORIES DU CHANGEMENT ${ }^{10}$}

Si concevoir et utiliser les théories du changement peut paraitre compliqué, c'est uniquement parce qu'une " théorie du changement " nest pas un objet en soi, un peu comme le concept d' " évaluation » peut représenter bien des choses différentes selon les situations. Les théories du changement :

- évoluent au fil du temps;

- ont des objectifs différents;

- doivent prendre en compte l'incertitude et la non-linéarité;

- peuvent être formulées ex ante et ex post.

Il existe différentes ressources pour guider l'élaboration de théories du changement. Vogel (2012a) et Barnett et Gregorowski (2013) abordent les théories du changement sur la question de l'influence de la recherche. Le vaste site Web 
www.theoryofchange.org traite en détail des théories du changement et propose des références à plusieurs autres guides et documents pertinents.

Pour obtenir une robuste première version d'une théorie du changement, il est nécessaire de tester la logique d'intervention et les hypothèses à la lumière de résultats de recherche ou d'évaluation existants qui pourraient confirmer (ou non) la théorie de changement établie. Brousselle et Champagne (2011) et Kautto et Silila (2005) discutent de cette mise à l'épreuve de la théorie de changement et suggèrent que cette étape est importante avant d'évaluer si l'intervention a donné des résultats. Ce type d'analyse permettrait d'identifier des points faibles dans les idées et hypothèses initiales sur le fonctionnement anticipé de l'intervention. Comme nous l'avons noté au départ, une théorie du changement peut aussi servir de cadre pour la conception de l'intervention, l'élaboration d'un système de monitorage et le développement d'un plan d'évaluation.

Lors de l'élaboration de chaînes d'effets et de théories du changement, il faut garder en tête plusieurs éléments :

- Il est probablement préférable de les établir de façon participative, bien que ce ne soit pas toujours possible.

- Dans un processus participatif, on peut soit commencer avec une page blanche et construire le modèle en sollicitant de différents points de vue, soit (ce qui est peut-être plus efficace) tracer d'abord, en petit comité, une ébauche de chaîne d'effets ou de théories du changement qui servira ensuite de base pour la discussion.

- Plus d'une version de la théorie du changement d'une intervention peut émerger des discussions avec les parties prenantes (Hansen et Vedung, 2010; Weiss, 1997). Il peut alors s'avérer utile de tester la vraissemblance de chacune des versions.

- Il est important d'inclure, de façon explicite ou implicite, tous les éléments de la théorie du changement.

- Le développement des chaînes d'effets et des théories du changement doit être considéré comme un processus; elles évoluent au fur et à mesure des connaissances acquises.

- Il faut accepter de produire une chaîne des effets ou une théorie du changement « acceptable » plutôt qu'une version parfaite.

- Les changements dans les capacités et de comportement sont souvent cruciaux dans les interventions.

- Autant que possible, les chaînes d'effets et les théories du changement devraient se fonder sur des recherches antérieures en plus du point de vue des parties prenantes.

- L'imbrication des chaînes d'effets et des théories du changement ou de la portée est un procédé très utile. Les éléments imbriqués seélaborent autour de certaines stratégies ou groupes cibles de l'intervention.

- Les théories du changement peuvent être représentées de diverses façons et à divers degrés de détail. 
- Des chaînes d'effets et des théories du changement génériques peuvent être très utiles pour représenter les principaux éléments d'une même intervention implantée dans différents contextes.

\section{CONCLUSION}

Établir des théories du changement crédibles est essentiel pour la conduite d'évaluations fondées sur la théorie (theory-based evaluations). Les modèles présentés ici se veulent assez adaptables pour s'appliquer à un large éventail d'interventions. Dans cet article, j’avance que les modèles de théorie du changement illustrés de façon générique aux figures 1 et 3 sont « utiles ", pour plusieurs raisons :

- Ces modèles donnent souvent une représentation " acceptable » d'une théorie du changement sans verser dans lexcès de complexité. Ils jettent les bases pour l'élaboration d'histoires de la performance (Mayne, 2004).

- Ces modèles évitent délibérément d'utiliser la terminologie d'extrants, d'effets à court terme, d'effets intermédiaires et à long terme. Cette classification n'a pas de signification intrinsecte, n'est pas utile pour construire une théorie de changement et conduit trop souvent à des débats stériles. C'est davantage la séquence d’événement qui est importante. Les produits de l'intervention, la portée, les changements dans les capacités, de comportement, etc. tels qu'indiqués dans les figures 1 et 3 portent un contenu et offrent une meilleure structure analytique pour lélaboration d'une théorie de changement.

- Les hypothèses sur les liens de causalité sont importantes pour décrire ce qui est nécessaire pour que le lien fonctionne; elles sont centrales dans la théorie du changement.

- Le recours à des boites pour représenter les hypothèses sur les liens de causalité simplifie la représentation et évite la prolifération des cases et des flèches.

- Il est souvent possible de simplifier un peu le modèle de théorie du changement en supprimant une case " résultat » et en incluant cet élément sous forme d'hypothèse.

- On peut simplifier les théories du changement en identifiant des théories imbriquées.

- Le modèle qui comprend les hypothèses sur les facteurs de soutien fait directement le lien entre le faisceau de causalité et les causes contributives, offrant une base rigoureuse pour une discussion sur la causalité.

\section{NOTES}

1 Le terme "modèle logique " est parfois utilisé comme synonyme de «théorie du programme » ou de « théorie du changement » (Funnell et Rogers, 2011), mais souvent, il 
est une simple représentation de la chaîne causale. Ainsi, par exemple, le gouvernement du Canada définit « modèle logique » comme étant une «[i]llustration de la relation de cause à effet, ou lien logique, entre les activités, les intrants, les extrants et les résultats d'une politique, d'un programme, ou d'une initiative, par exemple la chaîne des résultats» (Secrétariat du Conseil du Trésor, 2012).

2 Les principales corrections apportées visaient à simplifier la représentation du modèle en éliminant les références explicites aux « risques », aux " autres facteurs explicatifs » et aux « incitatifs » et à mentionner explicitement les résultats imprévus.

3 Le savoir relève de l'information apprise ou des conseils suivis; les attitudes ont trait aux croyances, aux opinions, aux sentiments ou aux points de vue; les compétences renvoient aux habiletés mentales et physiques nécessaires à l'adoption de pratiques nouvelles ou de substitution; les aspirations englobent les ambitions, les espoirs, les objectifs ou les désirs. Adapté de Bennett et Rockwell (1995, p. 6).

4 Le «bien-être » est le terme générique utilisé ici pour décrire le résultat visé. On pourrait aussi parler $\mathrm{du}$ « mode de vie».

5 Dans des articles précédents, javais souvent inclus explicitement les « risques » dans les cases des hypothèses et souligné que certaines hypothèses se comprennent et sénoncent plus facilement sous forme de risques. Bien que parfois utile, cette technique a toutefois tendance à encombrer les cases.

6 Les théories de la portée imbriquées qui sont mentionnées dans la figure 2 seront abordées plus loin.

7 Lacronyme INNNS (INUS en anglais) représente une partie Insuffisante, mais Nécessaire d'une condition elle-même Non Nécessaire mais Suffisante pour que l'effet se produise (Mackie, 1974).

8 La figure 3 illustre un type d'intervention compliquée et complexe comportant des activités de base et de soutien. Un autre type d'intervention (non illustré), l'intervention multicomposante suffisante, se compose d'un certain nombre d'activités plutôt séparées et distinctes qui, mises ensemble, sont censées produire une amélioration du bien-être. Dans ce cas, les composantes seraient des théories du changement imbriquées dans une théorie du changement générale plus vaste.

9 Les théories de la portée font l'objet d'une analyse dans Mayne (2014).

10 Les points de la présente section sont développés plus avant dans Mayne et Johnson (2015).

\section{RÉFÉRENCES}

Barnett, C., et R. Gregorowski (2013). Learning about theories of change for the monitoring and evaluation of research uptake (IDS Practice Paper in Brief, ILT Brief 14: Institute of Development Studies), http://opendocs.ids.ac.uk/opendocs/bitstream/ handle/123456789/2995/PP\%20InBrief\%2014\%20FINAL.pdf;jsessionid=4E0FEAA E919534B1B2A65F654E121023? sequence $=1$.

Bennett, C., et K. Rockwell (1995). Targeting outcomes for programs: A hierarchy for targeting outcomes and evaluating their acheivement (University of Nebraska), ftp:// bsesrv214.bse.vt.edu/grisso/Program_Logic/Targeting_Outcome_Programming.pdf.

Bickman, L. (dir.) (1987). Using program theory in evaluation (New Directions for Evaluation, $\left.n^{\circ} 35\right)$, San Francisco, Jossey-Bass. 
Brousselle, A., et F. Champagne (2011). Program theory evaluation: Logic analysis. Evaluation and Program Planning, vol. 34, p. 69-78.

Cartwright, N., et J. Hardie (2012). Evidence-based policy: Doing it better. A practical guide to predicting if a policy will work for you. Oxford, Oxford University Press, http:// dx.doi.org/10.1093/acprof:osobl/9780199841608.001.0001.

Chen, H. T. (2015). Practical program evaluation: Theory-driven evaluation and the integrated evaluation perspective ( $2^{\mathrm{e}}$ éd.), Thousand Oaks, Sage.

Darnton, A. (2008). Practical guide: An overview of behaviour change models and their uses, http://www.civilservice.gov.uk/wp-content/uploads/2011/09/Behaviour-change_ practical_guide_tcm6-9696.pdf.

Funnell, S., et P. Rogers (2011). Purposeful program theory: Effective use of theories of change and logic models, San Francisco, Jossey-Bass.

Gatersleben, B., et C. Vlek (1998). Household consumption, quality of life and environmental impacts, dans K. Noorman et A. Schoot-Uiterkamp (dir.), Green households? Domestic consumers, environment and sustainability, Londres, Earthscan, p. 141-183.

Groupe des Nations Unies pour le développement (2011). Results-based management handbook: Harmonizing RBM concepts and approaches for improved development results at country level, https://undg.org/wp-content/uploads/2015/01/UNDG-RBMHandbook-2012.pdf.

Hansen, M. B., et E. Vedung (2010). Theory-based stakeholder evaluation. American Journal of Evaluation, vol. 31, nº 3, p. 295-313, http://dx.doi.org/10.1177/1098214010366174.

James, C. (2011). Theory of change review (Report commissioned by Comic Relief), http:// mande.co.uk/blog/wp-content/uploads/2012/03/2012-Comic-Relief-Theory-ofChange-Review-FINAL.pdf.

Johnson, N., H. Guedenet et A. Saltzman (2014). What will it take for biofortification to have impact on the ground? Theories of change for three crop-country combinations (IFPRI discussion paper), Washington, International Food Policy Research Institute, http:// ebrary.ifpri.org/cdm/singleitem/collection/p15738coll2/id/129089/rec/1.

Johnson, N., J. Mayne, D. Grace et A. Wyatt (2015). How will training traders contribute to improved food safety in informal markets for meat and milk? A theory of change analysis (IFPRI discussion paper 1451). Washington: International Food Policy Research Institute, http://ebrary.ifpri.org/cdm/ref/collection/p15738coll2/id/129293.

Kautto, P., et J. Silila (2005). Recently introduced policy instruments and intervention theories, Evaluation, vol. 11, no 1, p. 55-68.

Ling, T. (2012). Evaluating complex and unfolding interventions in real time, Evaluation, vol. 18, nº 1, p. 79-91, http://dx.doi.org/10.1177/1356389011429629.

Mackie, J. L. (1974). The cement of the universe: A study of causation, Oxford, Oxford University Press.

Mahoney, J. (2008). Toward a unified theory of causality. Comparative Political Studies, vol. $41, \mathrm{n}^{\text {os }} 4-5$, p. 412-436, http://dx.doi.org/10.1177/0010414007313115.

Mayne, J. (2004). Reporting on outcomes: Setting performance expectations and telling performance stories, Revue canadienne dévaluation de programme, vol. $19, \mathrm{n}^{\circ} 1$, p. 31-60. 
Mayne, J. (2008). Contribution analysis: An approach to exploring cause and effect, (ILAC Brief No. 16). The Institutional Learning and Change Initiative, http://www.cgiar-ilac. org/files/publications/briefs/ILAC_Brief16_Contribution_Analysis.pdf.

Mayne, J. (2011). Contribution analysis: Addressing cause and effect, dans R. Schwartz, K. Forss et M. Marra (dir.), Evaluating the complex, New Brunswick (NJ), Transaction, p. 53-96.

Mayne, J. (2012). Contribution analysis: Coming of age? Evaluation, vol. 18, n 3, p. 270 280, http://dx.doi.org/10.1177/1356389012451663.

Mayne, J. (2014). Using theories of reach to enhance equity considerations in evaluation, dans K. Forss et M. Marra (dir.), Speaking justice to power: Ethical and methodological challenges for evaluators, New Brunswick (NJ), Transaction, p. 133-152.

Mayne, J., et N. Johnson (2015). Using theories of change in the Agriculture for Nutrition and Health CGIAR research program, Evaluation, vol. 21, n 4 , p. 407-428.

Montague, S., et N. Porteous (2013). The case for including reach as a key element of program theory, Evaluation and Program Planning, vol. 36, n 1, p. 177-183, http://dx.doi. org/10.1016/j.evalprogplan.2012.03.005.

Morra Imas, L. G., et R. C. Rist (2009). The road to results: Designing and conducting effective development evaluations, Washington, World Bank.

Patton, M. Q. (2008). Utilization-focused evaluation (4e éd.), Thousand Oaks, Sage.

Rogers, P. (2008). Using programme theory to evaluate complicated and complex aspects of interventions, Evaluation, vol. 14, $\mathrm{n}^{\circ} 1$, p. 29-48, http://dx.doi. org/10.1177/1356389007084674.

Rogers, P. (2011). Implications of complicated and complex characteristics for key tasks in evaluation, dans K. Forss, M. Marra et R. Schwartz (dir.), Evaluating the complex: Attribution, contribution and beyond, New Brunswick (NJ), Transaction, p. 33-52.

Rossi, P. H., M. W. Lipsey et H. E. Freeman (2004). Evaluation: A systematic approach (7 éd.), Thousand Oaks, Sage.

Sanderson, I. (2000). Evaluation in complex policy systems. Evaluation, vol. 6, no 4, p. 433-454, http://dx.doi.org/10.1177/13563890022209415.

Secrétariat du Conseil du Trésor (2012). Lexique de la gestion axée sur les résultats, Ottawa, SCT, http://www.tbs-sct.gc.ca/hgw-cgf/oversight-surveillance/ae-ve/cee/pubs/ lex-fra.asp.

Stein, D., et C. Valters (2012). Understanding "theory of change" in international development: A review of existing knowledge, Asian Institute and the Justice and Security Research Programme, http://www.theoryofchange.org/wp-content/uploads/toco_library/pdf/UNDERSTANDINGTHEORYOFChangeSteinValtersPN.pdf.

Suchman, E. D. (1967). Evaluative research: Principles and practice in public service and social action programs, New York, Russell Sage Foundation.

Vogel, I. (2012a). ESPA guide to working with theory of change for research projects, Ecosystem Services for Alleviation of Poverty, http://www.espa.ac.uk/files/espa/ESPATheory-of-Change-Manual-FINAL.pdf. 
Vogel, I. (2012b). Review of the use of "theory of change" in international development, Department for International Development, http://www.oxfamblogs.org/fp2p/wpcontent/uploads/DFID-ToC-Review_VogelV4.pdf.

Weiss, C. H. (1997). How can theory-based evaluation make greater headway? Evaluation Review, vol. 21, no 4, p. 501-524, http://dx.doi.org/10.1177/0193841X9702100405.

White, H. (2009). Theory-based impact evaluation: Principles and practice (Working Paper 3), International Initiative for Impact Evaluation (3ie), http://www.3ieimpact.org/ admin/pdfs_papers/48.pdf, http://dx.doi.org/10.1080/19439340903114628.

\section{PRÉSENTATION D'AUTEUR}

John Mayne, Ph. D., est un conseiller indépendant en analyse de la performance dans le secteur public. Depuis 11 ans, ses travaux portent principalement sur l'évaluation et la gestion axée sur les résultats dans le domaine du développement international. Il a collaboré avec des organismes publics, des ONG et des organisations internationales. Auteur de nombreux articles et rapports, notamment sur l'analyse de la contribution, il a assuré la codirection de huit ouvrages sur l'évaluation de programme et l'analyse de la performance. En 1989 et en 1995, il a reçu le Prix pour la contribution à lévaluation au Canada de la Société canadienne dévaluation. En 2006, il a été nommé membre titulaire de la Société canadienne dévaluation. Ses recherches actuelles portent sur l'évaluation des impacts, les théories du changement et la question de l'attribution. 